\title{
OSTEOCYTE DEFICIENCY IN HIP FRACTURES
}

Jesús Delgado-Calle ${ }^{1}$, Jana Arozamena ${ }^{1}$, Raúl García-Renedo ${ }^{2}$, Carmen García-Ibarbia ${ }^{1}$, María A. Pascual-Carra ${ }^{2}$, Jesús González-Macías ${ }^{1}$ and José A. Riancho ${ }^{1}$

${ }^{1}$ Department of Internal Medicine. Hospital U.M. Valdecilla-IFIMAV-University of Cantabria. Santander, Spain

${ }^{2}$ Department of Orthopaedic Surgery and Traumatology. Hospital U.M. Valdecilla. Santander, Spain.

Running title: Osteocyte function and fractures

Correspondence and reprint requests:

José A. Riancho

Department of Internal Medicine

Hospital U.M. Valdecilla, University of Cantabria

Santander 39008, Spain

Phone: 34-942201990

Fax: 34-942201695

Email: rianchoj@unican.es 


\section{ABSTRACT}

Purpose. Osteocytes play a central role in the regulation of bone remodeling. The aim of this study was to explore osteocyte function, and particularly the expression of SOST, a Wnt inhibitor, in patients with hip fractures.

Methods. Serum sclerostin levels were measured by ELISA. The expression of several osteocytic genes was studied by quantitative PCR in trabecular samples of the femoral head of patients with hip fractures, hip osteoarthritis and controls. The presence of sclerostin protein and activated caspase 3 was revealed by immunostaining

Results. There were no significant differences in serum sclerostin between the three groups. Patients with fractures have fewer lacunae occupied by osteocytes $(60 \pm 5 \%$ vs. $64 \pm 6 \%$ in controls, $\mathrm{p}=0.014$ ) and higher numbers of osteocytes expressing activated caspase 3 , a marker of apoptosis. The proportion of sclerostin-positive lacunae was lower in patients with fractures than in controls $\left(34 \pm 11 \%\right.$ vs. $\left.69 \pm 10 \%, p=2 \times 10^{-8}\right)$. The proportion of sclerostin-positive osteocytes was also lower in patients. RNA transcripts of SOST , FGF23 and PHEX were also less abundant in fractures than in control bones ( $\mathrm{p}=0.002,5 \times 10^{-6}$, and 0.04 , respectively). On the contrary, in patients with osteoarthritis, there was a decreased expression of SOST and FGF23, without differences in PHEX transcripts or osteocyte numbers.

Conclusions. Osteocyte activity is altered in patients with hip fractures, with increased osteocyte apoptosis and reduced osteocyte numbers, as well as decreased transcription of osteocytic genes. Therefore, these results suggest that an osteocyte deficiency may play a role in the propensity to hip fractures.

KEYWORDS: Sclerostin, FGF23, PHEX, osteocytes, osteoporosis. 


\section{INTRODUCTION}

After the seminal studies showing that gain-of-function and loss-of-function mutations of the Wnt co-receptor lipoprotein related peptide 5 (LRP5) are associated with marked changes of opposite direction in bone mass, the Wnt pathway has been recognized as an important bone anabolic stimulus, influencing bone remodelling and fracture repair [1;2]. Binding of Wnt ligands to their receptors induce the disassembly of the GSK3 complex that phosphorylates $\beta$ catenin. Since non-phosphorylated $\beta$-catenin is less prone to proteasome degradation, this results in increased $\beta$-catenin levels and translocation into the nucleus, where it modulates the transcription of target genes [3;4].

Osteocytes are the most abundant cells in bone. They derive from osteoblasts that become embedded within the bone matrix laid during the bone formation phase of bone remodelling. Although initially regarded as a passive process, it is now clear that the osteoblast-osteocyte transition is an organized process that includes specific changes in the gene expression pattern. Osteocytes are nowadays recognized as major players in the regulation of bone remodelling. They are considered as the cells transducing the effects of mechanical forces acting on bone, able to mark the skeletal areas where a new remodelling cycle is to be initiated, and with strong influence on bone formation [5-7]. In fact, osteocytes synthesize several molecules able to modulate osteoblast function, including prostaglandins, sclerostin and dickkopf 1 (DKK1). Sclerostin, the product of the SOST gene, and DKK1 are potent inhibitors of the Wnt pathway. We have previously reported that, in comparison with patients with osteoarthritis (OA), the expression of a number of genes in the Wnt pathway is reduced in bone samples from patients with osteoporotic hip fractures, suggesting that differences in Wnt activity may be involved in the opposite changes in bone mass typical of these disorders. However, we did not find significant differences in DKK1 expression between both groups of patients [8].

On the basis of those data, we hypothesized that an osteocyte dysfunction with increased expression of sclerostin could be involved in the reduced Wnt activity found in hip fractures. 
Therefore, in the present study we determined the expression of sclerostin and other genes characteristic of an osteocytic phenotype, as well as osteocyte distribution in patients with hip fractures, comparing the results with those in hip OA and control bones.

\section{MATERIALS AND METHODS}

\section{Bone samples}

Bone samples were obtained during hip replacement surgery, in patients over 50 years of age with hip fractures (Frx) or hip OA, who provided informed consent. Patients with secondary osteoporosis, fractures due to high-energy trauma or secondary OA were excluded. Control samples were obtained at autopsy of patients without known bone disorders. The study was approved by the Institutional Ethics Committee. Trabecular bone cylinders of the central part of the femoral head (thus avoiding the fractured and the subchondral regions) were obtained with a trephine, washed extensively in phosphate-buffered saline and either snap-frozen in liquid nitrogen and stored at $-70^{\circ}$ until RNA analysis, or processed for histology and immunohistochemistry (bones used for both procedures largely but incompletely overlapped, due to sample availability) .

\section{Quantitation of gene expression and sclerostin serum levels}

Bone samples from 18 patients with fractures (13 women and 5 men, mean age $82 \pm 5 \mathrm{yr}$ ), 21 with hip osteoarthritis (16 women and 5 men; age $72 \pm 7$ yr) and 10 controls (4 women and 6 men; age $79 \pm 6 \mathrm{yr})$ were used. RNA was isolated from bone samples by using Trizol, following manufacturer's instructions (Invitrogen, Carlsbad, CA, USA) and purified using the RNeasy kit (Qiagen, Hilden, Germany), as previously reported [9].

Aliquots of RNA were reverse-transcribed with the Superscript III kit (Invitrogen), using random hexamers as primers. Then, the expression of three genes mainly expressed by osteocytes (SOST/sclerostin, fibroblast growth factor 23 [FGF23], and phosphate regulating endopeptidase homolog, X-linked $[P H E X]$ ) was determined by real-time PCR using gene- 
specific primers and Taqman probes (Applied Biosystems, Foster City, CA, USA). The threshold cycle $(\mathrm{Ct})$ for each well was determined. The results were normalized to the expression of the housekeeping gene TATA box protein (TBP). Relative gene expression was calculated as $2^{-\mathrm{Ct}}$, where $\Delta \mathrm{Ct}$ is the difference between the gene of interest threshold cycle and the TBP threshold cycle.

Serum sclerostin levels were measured in patients with hip fractures $(n=16$, age $78 \pm 3 \mathrm{yr}$ ) or hip OA ( $\mathrm{n}=16$, age $76 \pm 4$ ), and in control healthy individuals without osteoporosis or other disorders affecting skeletal homeostasis $(\mathrm{n}=12$, age $78 \pm 3)$, using a commercial ELISA kit (Tecomedical, Sissach, Switzerland), with a sensitivity of $0.15 \mathrm{ng} / \mathrm{ml}$.

\section{$\underline{\text { Histology and immunostaining }}$}

Trabecular samples from the femoral heads of 21 patients with fractures (17 women, 4 men; mean age $82 \pm 9$ yr), 8 with hip OA ( 4 women, 4 men; age $75 \pm 4$ yr) and 8 controls ( 4 women, 4 men; age $79 \pm 6 \mathrm{yr}$ ) were fixed in $4 \%$ formalin for 3 days, decalcified with $20 \%$ EDTA for 3 weeks, and embedded in paraffin. Four-micron thick sections were cut and mounted on gelatine-coated slides. Prior to staining, slides were heated for 20 minutes at $60^{\circ} \mathrm{C}$, hydrated through graded alcohols, immersed in 50mM sodium citrate buffer for 15 minutes for antigen unmasking, and incubated in $0.3 \%$ hydrogen peroxide in methanol for 30 minutes to quench the endogenous peroxidase activity. After blocking non-specific binding with normal goat serum, the slides were incubated overnight with a rabbit polyclonal anti-sclerostin antibody (Santa Cruz, Heidelberg, Germany), diluted 1/200, followed by a secondary biotinylated anti rabbit antibody and then with avidin-biotinylated horseradish peroxidase and DAB substrate, following manufacturer's instructions (ABC Peroxidase Rabbit Staining kit; Pierce, Rockford, IL, USA). Slides were counterstained with haematoxylin for 1 minute prior to mounting and examined at 400x magnification. Osteocytic lacunae (raw and normalized to the trabecular bone area), as well as the presence or absence of osteocytes and sclerostin staining were counted in 16 random fields. The distance from the osteocytic lacunae to the closest trabecular 
surface was measured with a scale grid. Apoptotic osteocytes were identified by using a similar protocol with an anti-activated caspase 3 antibody (R\&D Systems, Minneapolis, MN, USA) diluted 1/100. Appropriate negative control slides without the primary antibodies were prepared. The proportion of lacunae occupied by osteocytes, and the proportions of lacunae and osteocytes with staining for sclerostin and activated caspase were calculated. All analyses were done blindly, without prior knowledge of the group of the sample.

\section{$\underline{\text { Statistical analyses }}$}

The statistical significance of the differences in gene expression was tested by the KruskalWallis test, followed by pair-wise posthoc Mann-Whitney tests, if the global test was significant. To compare the results of the histological and immunohistochemical studies, we used an ANOVA test, followed by t-test if significant results were found by ANOVA, with or without assumption of equal variances according to the Levene test. Prism and SPSS packages were used in the analyses. All reported p-values are two-tailed.

\section{RESULTS}

$\underline{\text { Serum sclerostin levels and gene expression in bone }}$

All individuals had detectable serum sclerostin levels. However, we could not demonstrate the hypothesized increase in serum sclerostin in patients with fractures. In fact, in comparison with patients with $\mathrm{OA}$ and control individuals, patients with fractures showed a non significant trend to lower sclerostin levels. The mean $( \pm \mathrm{SD})$ values were $0.78 \pm 0.17,0.80 \pm 0.46$ and $0.71 \pm 0.30 \mathrm{ng} / \mathrm{ml}$, in control individuals, patients with $\mathrm{OA}$ and patients with fractures, respectively (fig. 1). At the bone tissue level there were significant differences in sclerostin gene expression across the three groups of individuals $(\mathrm{p}=0.020)$. In pair-wise comparisons, SOST expression was lower in patients with fractures than in controls $(\mathrm{p}=0.002)$, whereas similar levels were found in fracture and OA samples ( $\mathrm{p}=0.69)$ (fig. 2). 
Given the well-known inhibitory effect of sclerostin on bone formation, those results were unexpected. Therefore, we explored the transcript abundance of other genes typically expressed in osteocytes, such as FGF23 and PHEX (fig. 2). We also found marked differences in the transcript levels of these genes across the groups of patients studied ( $p=0.00004$ and $\mathrm{p}=0.035$, for FGF23 and PHEX, respectively). Patients with fractures had lower FGF23 expression levels than either controls or patients with hip OA $(\mathrm{p}=0.000005$ and 0.003 , respectively). The average $P H E X$ transcript abundance was also lower in patients with fractures than in the other groups ( $p=0.04$ versus controls, $p=0.02$ versus $O A$ ).

There was a direct correlation between FGF23 and PHEX expression in the three study groups $(p=0.0001)$. However, SOST expression did not correlate with FGF23 or PHEX expression.

\section{Osteocyte distribution and immunohistochemistry}

The above-mentioned results suggested the existence of an overall osteocyte dysfunction in patients with fractures. Therefore, we analyzed osteocyte numbers and sclerostin immunoreactivity in bone biopsies to elucidate whether it was due to a decreased number of osteocytes or to a qualitative defect.

There were no differences in the number of osteocytic lacunae, adjusted by trabecular bone surface, between the three groups studied $(\mathrm{p}=0.2)$. After immunostaining of trabecular bone samples, sclerostin was detected only in osteocytic lacunae and along osteocytic canaliculi. Sclerostin-positive lacunae tended to be located in deeper layers of bone than sclerostinnegative lacunae. This was a consistent and highly significant finding in all groups, but without differences in the depth of sclerostin-positive lacunae across groups (fig. 3).

The proportion of sclerostin-positive lacunae showed wide individual variations but was much lower in patients with fractures than in controls $\left(34 \pm 11 \%\right.$ vs. $\left.69 \pm 10 \%, p=2 \times 10^{-8}\right)$. It also showed a non-significant trend for lower values in fractures than in OA $(34 \pm 11 \%$ vs. $44 \pm 21$ $\%, p=0.2$ ) (fig. 4). Sclerostin immunostaining was found in some osteocyte-containing lacunae and also in some empty lacunae. When only the occupied lacunae were considered, there were 
also marked differences across groups: the proportion of sclerostin-positive osteocytes was $35 \pm 14 \%$ in patients with fractures and $69 \pm 10 \%$ in controls $\left(p=1 \times 10^{-6}\right)$

Patients with fractures had fewer occupied lacunae (i.e., lacunae with an osteocyte inside). The proportion of occupied lacunae was $60 \pm 5 \%$ in fractures, $64 \pm 6 \%$ in controls ( $p=0.014$ vs. fractures) and $73 \pm 9 \%$ in OA ( $\mathrm{p}=0.005$ vs. fractures). This was associated with more frequent activated caspase 3 immunostaining, a marker of apoptosis, in fractures (fig 4). The proportion of caspase-positive lacunae was $53 \pm 8 \%$ in fractures, $41 \pm 6 \%$ in controls $(p=0.002)$ and $40 \pm 6$ in $\mathrm{OA}(\mathrm{p}=0.001$ vs. fractures). In fact, there was an inverse correlation between the proportion of occupied lacunae and those with caspase-positive staining $(r=-0.46, p=0.015)$.

The average time between fracture and surgery was 4 days (range 0-9). There were no correlations between the surgery delay and the proportions of empty lacunae, sclerostinpositive lacunae or caspase-positive osteocytes (data not shown). No significant associations existed with sex or age. Therefore, similar results were obtained in the sex-stratified analyses (not shown).

The main differences in tissue-level parameters across the groups are summarized in figure 5.

\section{DISCUSSION}

The activation of the Wnt pathway is an important stimulus for bone formation. We have previously reported a reduced expression of several genes of the Wnt pathway in bone from patients with hip fractures [8], but the mechanisms involved remained unclear. Sclerostin is a secreted protein that binds to LRP5/6 and prevents the effective interaction of this co-receptor

with its ligands of the Wnt family [10-12]. Its role was emphasized by the identification of SOST (the gene encoding sclerostin) mutations in patients with Van Buchem disease and sclerosteosis, disorders characterized by a markedly increased bone mass [13]. In line with this idea, neutralizing anti-sclerostin antibodies have been shown to increase bone formation in experimental models of osteoporosis [14], as well as in short-term studies in humans [15]. In 
this study we confirmed that sclerostin is abundantly expressed in human bone, where it was detected in osteocytes and osteocytic lacunae, but not in osteoblasts or other cells.

Since sclerostin is a potent inhibitor of Wnt signaling, we hypothesized that an increased expression of sclerostin could explain the reduced Wnt activity in fractures. However, the experimental results did not support this hypothesis. In fact, patients with fractures had sclerostin serum levels similar to control individuals and patients with hip OA. Moreover, SOST expression and the proportion of sclerostin-positive lacunae were lower in bone samples from patients with fractures. This result appeared counterintuitive at first sight and suggested the existence of an abnormal osteocyte activity in fractures. In fact, when we analyzed other genes typically expressed in osteocytes, such as PHEX and FGF23, they were also found to be down-regulated in fractures. Osteocytes derive from osteoblasts, which become embedded into bone matrix when new bone is laid down and stay there inside the osteocytic lacunae. We found similar lacunae density in the three groups of subjects, suggesting that similar numbers of osteocytes were generated during the bone formation phase. However, the proportion of empty lacunae was higher in fractures. Since the lacunae were initially formed around an osteocyte, it suggested that osteocyte death was more frequent in fracture samples. Indeed, we found a higher proportion of lacunae staining positively for activated caspase 3 , which is consistent with increased apoptosis of osteocytes.

Loading experiments in rats have shown that osteocyte apoptosis takes place in regions of bone with microcracks and is necessary to initiate intracortical bone remodelling in response to fatigue microdamage $[16 ; 17]$. Osteocyte apoptosis, which progresses over several days, has also been observed in the vicinity of macroscopic bone lesions [18]. However, apoptosis occurring post-fracture is unlikely to explain the differences between patients and controls. First, Power et al. have recently reported that sclerostin expression may be locally decreased in osteocytes within $0.5 \mathrm{~mm}$ of a bone fracture, but is not altered in those located more distant [19]. We did not include tissue close to the fracture site, but used bone samples more than 5 
mm away of fracture. Second, we did not find any relationship between time elapsed since fracture until surgery and gene expression or osteocyte apoptosis.

Osteocytes appear to play a critical role in mechanotransduction, and are involved in the anabolic response of bone to physical forces [6;20]. In fact, osteocytes have been postulated as the central "metabolostat", integrating and orchestrating a response to different mechanical and humoral factors acting on bone [21]. In studies with a transgenic mouse that expressed the receptor for diphtheria toxin in osteocytes, Tatsumi et al showed that the ablation of osteocytes caused an acute increase in bone resorption and cortical microfractures [20], suggesting that osteocyte deficiency may result in osteoporosis. Therefore, it is tempting to speculate that the increased osteocyte apoptosis that we observed in patients with hip fractures actually played a role in determining fracture risk. This is in line with a recent report showing a increased number of empty lacunae in a sheep model of osteoporosis [22].

However, the increased apoptosis of osteocytes was not the only factor contributing to the reduced expression of osteocytic genes in fractures. In fact, the proportion of osteocyteoccupied lacunae which stained positively for sclerostin was lower in fractures than in controls. Furthermore, while the proportion of empty lacunae was about $10 \%$ higher in patients with fractures than in controls, the average SOST expression was reduced by more than $50 \%$, and the expression of FGF23 and PHEX was decreased even further, down to less than $5 \%$ and $30 \%$ of control values, respectively. Therefore, we can speculate about the existence of some factors inhibiting the expression of these genes in the osteocytes of patients with fractures. They might include intrinsic factors, such as genetic or epigenetic signatures, or extrinsic factors, such as hormones, paracrine soluble factors and matrix constituents. In this regard, it is interesting to note that the degree of calcification and other characteristics of the matrix surrounding osteocytic lacunae have been suggested to modulate the osteoblast-osteocyte transition [23]. We explored whether osteocyte distribution within bone, and particularly, the depth of the location of osteocytic lacunae, influenced sclerostin expression. That appeared to 
be the case. Consistently with other reports $[7 ; 11 ; 23]$, sclerostin-positive lacunae tended to be located in deeper bone layers than sclerostin-negative lacunae. As osteocytes in deeper lacunae are probably older, and deep bone layers are more mineralized than the superficial ones, it suggests that a long time and/or a well mineralized matrix are needed for these cells to fully differentiate and acquire the ability to produce sclerostin. However, no differences in osteocyte depth distribution were found between the three groups of subjects.

Mechanical stimuli and PTH have been shown to inhibit sclerostin synthesis in vitro and in vivo [24-28]. Given the reduced bone mass and thinner trabeculae of patients with fragility fractures, it could be speculated that each individual osteocyte may be exposed to a higher mechanical stress in patients destined to sustain a hip fracture, which in turn could tend to decrease sclerostin expression. Studies combining microstructural and gene expression analyses could confirm or refute this contention, but it is consistent with studies showing increased levels of sclerostin in patients with high bone mass [29]. Vitamin D insufficiency and increased levels of PTH are common in patients with hip fractures [30], and would tend to decrease sclerostin expression. Unfortunately, we did not analyze serum PTH levels and therefore do not know if they were correlated with sclerostin circulating levels or expression, as suggested in other studies [31;32]. Although we found striking between-group differences in bone sclerostin at the mRNA and protein levels, there were no significant differences in circulating sclerostin. Therefore, serum levels do not appear to reflect adequately the bone microenvironment situation. In fact, counterintuitive positive associations between bone mineral density and serum sclerostin levels have been reported in several studies [32;33]. In order to explain these unexpected results, it should be realized that serum levels are the product of two factors: on the one hand, the expression level at each osteocyte; on the other hand, the total number of osteocytes, which is generally associated with the bone tissue mass of the individual. Therefore, osteoporotic patients with a low bone mass may have lower 
number of osteocytes and consequently they may show a trend to lower circulating levels of osteocyte-derived factors.

FGF23 expression was markedly decreased in patients with hip fractures. Its influence on skeletal homeostasis is unclear. It has been shown that PTH stimulates FGF23 expression in osteocytes, by Wnt-dependent and independent mechanisms. FGF23, in turn, may act on the parathyroids inhibiting the secretion of PTH [34;35]. Therefore, in theory the reduced expression of FGF23 could contribute to increase PTH levels in patients with hip fractures.

Our study has some limitations. First, we studied trabecular bone, which may behave differently from cortical bone. Nevertheless, Power et al recently studied sclerostin expression in the femoral neck cortex and found a non-significant trend to higher sclerostin-negative lacunae in hip fractures than in hip osteoarthritis [36]. Second, although our results do not give support for the hypothesis of an increased sclerostin expression in hip fractures, a single time point study like this cannot exclude the possibility that previous exposure to high local levels of sclerostin may have played a role in increasing bone fragility. Third, we studied femoral heads of Caucasian individuals and do not have data about expression levels in other skeletal regions, but some reports suggest that there may be subtle and incompletely elucidated differences in osteocyte status depending on the bone region studied, as well as on the ethnic background of the individuals [37;38]. In any case, our results are in line with a recent report showing a reduced sclerostin expression in iliac crest biopsies of male patients with idiopathic osteoporosis [39]. Sex and age distribution was somewhat different across the study groups, reflecting the epidemiological differences between fractures and OA. Although patients with fractures tended to be older than those with OA, age difference did not appear to influence the results because we found no correlation between age and sclerostin expression in either group. Mirza et al recently measured serum sclerostin levels in women and found no correlation with age either [31]. On the other hand, we did not find significant sex-related differences in the parameters studied. 
Interestingly, although osteocyte numbers were not decreased in patients with hip OA, SOST and FGF23 (but not PHEX) expression was lower in OA than in controls. These results are in line with a recent report by Appel et al [40] who showed a decreased proportion of sclerostinpositive osteocytes in the spinal joints of patients with OA. Although unproved, it can be speculated that a decreased expression of SOST might play a role in the local bone formation responsible for the osteophytes which typically appear in OA.

In summary, this study shows that osteocyte activity is altered in patients with hip fractures, with increased osteocyte apoptosis and reduced osteocyte numbers, as well as decreased transcription of several osteocyte-derived bone-regulating genes. Overall, these results suggest that an osteocyte deficiency may play a role in hip fractures. 


\section{ACKNOWLEDGEMENTS}

We acknowledge the excellent technical assistance of Carolina Sañudo. We thank

Tecomedical Inc. for providing ELISA the kits to measure serum sclerostin.

Supported by a grant from Instituto de Salud Carlos III/ Fondo de Investigaciones Sanitarias (FIS 09/0539). JD-C is recipient of a grant from IFIMAV 


\section{CONFLICTS OF INTEREST}

JDC, JGM and JAR have received research grants, travel grants or lecture fees from Amgen. 


\section{REFERENCES}

1. Komatsu DE, Mary MN, Schroeder RJ, Robling AG, Turner CH, Warden SJ (2010) Modulation of Wnt signaling influences fracture repair. J Orthop.Res 28:928-936

2. Moester MJ, Papapoulos SE, Lowik CW, van Bezooijen RL (2010) Sclerostin: current knowledge and future perspectives. Calcif.Tissue Int 87:99-107

3. Gaur T, Lengner CJ, Hovhannisyan H, Bhat RA, Bodine PV, Komm BS, Javed A, van Wijnen AJ, Stein JL, Stein GS, Lian JB (2005) Canonical WNT signaling promotes osteogenesis by directly stimulating Runx2 gene expression. J.Biol.Chem. 280:3313233140

4. Johnson ML, Harnish K, Nusse R, Van Hul W (2004) LRP5 and Wnt signaling: a union made for bone. J.Bone Miner.Res. 19:1749-1757

5. Bonewald LF (2011) The amazing osteocyte. J.Bone Miner.Res. 26:229-238

6. Rochefort GY, Pallu S, Benhamou CL (2010) Osteocyte: the unrecognized side of bone tissue. Osteoporos.Int. 21:1457-1469

7. Dallas SL, Bonewald LF (2010) Dynamics of the transition from osteoblast to osteocyte. Ann.N.Y.Acad.Sci. 1192:437-443

8. Velasco J, Zarrabeitia MT, Prieto JR, Perez-Castrillon JL, Perez-Aguilar MD, PerezNunez MI, Sanudo C, Hernandez-Elena J, Calvo I, Ortiz F, Gonzalez-Macias J, Riancho JA (2010) Wnt pathway genes in osteoporosis and osteoarthritis: differential expression and genetic association study. Osteoporos.Int 21:109-118

9. Hernandez JL, Garcés CM, Sumillera M, Fernandez-Aldasoro EV, Garcia-Ibarbia C, Ortiz JA, Arozamena J, Alonso MA, Riancho JA (2008) Aromatase expression in osteoarthritic and osteoporotic bone. Arthritis Rheum. 58:1696-1700

10. Li X, Zhang Y, Kang H, Liu W, Liu P, Zhang J, Harris SE, Wu D (2005) Sclerostin binds to LRP5/6 and antagonizes canonical Wnt signaling. J.Biol.Chem. 280:1988319887

11. Poole KE, van Bezooijen RL, Loveridge N, Hamersma H, Papapoulos SE, Lowik CW, Reeve J (2005) Sclerostin is a delayed secreted product of osteocytes that inhibits bone formation. FASEB J 19:1842-1844

12. Veverka V, Henry AJ, Slocombe PM, Ventom A, Mulloy B, Muskett FW, Muzylak M, Greenslade K, Moore A, Zhang L, Gong J, Qian X, Paszty C, Taylor RJ, Robinson MK, Carr MD (2009) Characterization of the structural features and interactions of 
sclerostin: molecular insight into a key regulator of Wnt-mediated bone formation. $\mathrm{J}$ Biol.Chem. 284:10890-10900

13. Loots GG, Kneissel M, Keller H, Baptist M, Chang J, Collette NM, Ovcharenko D, Plajzer-Frick I, Rubin EM (2005) Genomic deletion of a long-range bone enhancer misregulates sclerostin in Van Buchem disease. Genome Res 15:928-935

14. Li X, Ominsky MS, Warmington KS, Morony S, Gong J, Cao J, Gao Y, Shalhoub V, Tipton B, Haldankar R, Chen Q, Winters A, Boone T, Geng Z, Niu QT, Ke HZ, Kostenuik PJ, Simonet WS, Lacey DL, Paszty C (2009) Sclerostin antibody treatment increases bone formation, bone mass, and bone strength in a rat model of postmenopausal osteoporosis. J Bone Miner.Res 24:578-588

15. Padhi D, Jang G, Stouch B, Fang L, Posvar E (2011) Single-dose, placebo-controlled, randomized study of AMG 785, a sclerostin monoclonal antibody. J.Bone Miner.Res. 26:19-26

16. Cardoso L, Herman BC, Verborgt O, Laudier D, Majeska RJ, Schaffler MB (2009) Osteocyte apoptosis controls activation of intracortical resorption in response to bone fatigue. J.Bone Miner.Res. 24:597-605

17. Verborgt O, Gibson GJ, Schaffler MB (2000) Loss of osteocyte integrity in association with microdamage and bone remodeling after fatigue in vivo. J.Bone Miner.Res. 15:60-67

18. Clark WD, Smith EL, Linn KA, Paul-Murphy JR, Muir P, Cook ME (2005) Osteocyte apoptosis and osteoclast presence in chicken radii 0-4 days following osteotomy. Calcif.Tissue Int 77:327-336

19. Power J, Parker M, Papapoulos S, Van Bezooijen R, Reeve J, Loveridge N (2011) Osteocytic sclerostin expression is locally reduced at the site of femoral neck fracture. Bone 48 (suppl 2):S168 (abstract)

20. Tatsumi S, Ishii K, Amizuka N, Li M, Kobayashi T, Kohno K, Ito M, Takeshita S, Ikeda K (2007) Targeted ablation of osteocytes induces osteoporosis with defective mechanotransduction. Cell Metab 5:464-475

21. Watanabe K, Ikeda K (2010) Osteocytes in normal physiology and osteoporosis. Clin.Rev.Bone Miner.Metab. 8:224-232

22. Zarrinkalam MR, Mulaibrahimovic A, Atkins GJ, Moore RJ (2011) Changes in osteocyte density correspond with changes in osteoblast and osteoclast activity in an osteoporotic sheep model. Osteoporos.Int.

23. Irie K, Ejiri S, Sakakura Y, Shibui T, Yajima T (2008) Matrix mineralization as a trigger for osteocyte maturation. J Histochem.Cytochem. 56:561-567

24. Bellido T (2006) Downregulation of SOST/sclerostin by PTH: a novel mechanism of hormonal control of bone formation mediated by osteocytes. J Musculoskelet.Neuronal Interact. 6:358-359 
25. Leupin O, Kramer I, Collette NM, Loots GG, Natt F, Kneissel M, Keller H (2007) Control of the SOST bone enhancer by PTH using MEF2 transcription factors. J Bone Miner.Res 22:1957-1967

26. Silvestrini G, Ballanti P, Leopizzi M, Sebastiani M, Berni S, Di Vito M, Bonucci E (2007) Effects of intermittent parathyroid hormone (PTH) administration on SOST mRNA and protein in rat bone. J Mol.Histol. 38:261-269

27. Papanicolaou SE, Phipps RJ, Fyhrie DP, Genetos DC (2009) Modulation of sclerostin expression by mechanical loading and bone morphogenetic proteins in osteogenic cells. Biorheology 46:389-399

28. Robling AG, Niziolek PJ, Baldridge LA, Condon KW, Allen MR, Alam I, Mantila SM, Gluhak-Heinrich J, Bellido TM, Harris SE, Turner CH (2008) Mechanical stimulation of bone in vivo reduces osteocyte expression of Sost/sclerostin. J Biol.Chem. 283:5866-5875

29. Gregson C, Poole K, Steel C, Ayuk J, McCloskey E, Fraser W, Tobias J (2011) Elevated sclerostin levels in individuals with high bone mass. Bone 48 (suppl 2):S71S72 (abstract)

30. Fisher A, Srikusalanukul W, Davis M, Smith P (2010) Hip fracture type: important role of parathyroid hormone (PTH) response to hypovitaminosis D. Bone 47:400-407

31. Mirza FS, Padhi ID, Raisz LG, Lorenzo JA (2010) Serum Sclerostin Levels Negatively Correlate with Parathyroid Hormone Levels and Free Estrogen Index in Postmenopausal Women. J.Clin.Endocrinol.Metab. 95:1991-1997

32. Polyzos SA, Anastasilakis AD, Bratengeier C, Woloszczuk W, Papatheodorou A, Terpos E (2011) Serum sclerostin levels positively correlate with lumbar spinal bone mineral density in postmenopausal women-the six-month effect of risedronate and teriparatide. Osteoporos.Int

33. Modder UI, Hoey KA, Amin S, McCready LK, Achenbach SJ, Riggs BL, Melton LJ, III, Khosla S (2011) Relation of age, gender, and bone mass to circulating sclerostin levels in women and men. J Bone Miner.Res 26:373-379

34. Rhee Y, Bivi N, Farrow E, Lezcano V, Plotkin LI, White KE, Bellido T (2011) Parathyroid hormone receptor signaling in osteocytes increases the expression of fibroblast growth factor-23 in vitro and in vivo. Bone

35. Lavi-Moshayoff V, Wasserman G, Meir T, Silver J, Naveh-Many T (2010) PTH increases FGF23 gene expression and mediates the high-FGF23 levels of experimental kidney failure: a bone parathyroid feedback loop. Am.J Physiol Renal Physiol 299:F882-F889

36. Power J, Poole KE, Van Bezooijen R, Doube M, Caballero-Alias AM, Lowik C, Papapoulos S, Reeve J, Loveridge N (2010) Sclerostin and the regulation of bone formation: Effects in hip osteoarthritis and femoral neck fracture. J.Bone Miner.Res. $25: 1867-1876$ 
37. Qiu S, Rao DS, Palnitkar S, Parfitt AM (2003) Reduced iliac cancellous osteocyte density in patients with osteoporotic vertebral fracture. J.Bone Miner.Res. 18:16571663

38. Qiu S, Rao DS, Palnitkar S, Parfitt AM (2006) Differences in osteocyte and lacunar density between Black and White American women. Bone 38:130-135

39. Patsch JM, Kohler T, Berzlanovich A, Muschitz C, Bieglmayr C, Roschger P, Resch H, Pietschmann P (2011) Trabecular bone microstructure and local gene expression in iliac crest biopsies of men with idiopathic osteoporosis. J Bone Miner.Res

40. Appel H, Ruiz-Heiland G, Listing J, Zwerina J, Herrmann M, Mueller R, Haibel H, Baraliakos X, Hempfing A, Rudwaleit M, Sieper J, Schett G (2009) Altered skeletal expression of sclerostin and its link to radiographic progression in ankylosing spondylitis. Arthritis Rheum. 60:3257-3262 


\section{FIGURE LEGENDS}

Fig 1. Sclerostin serum levels measured by ELISA. Individual and mean values in each group. Frx, fractures; OA, osteoarthritis.

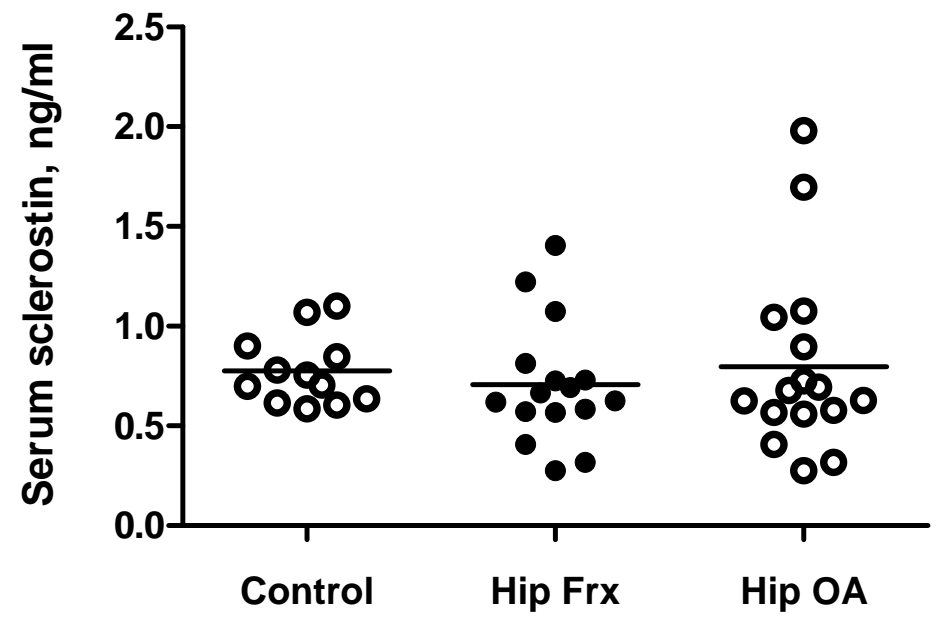


Fig, 2. Relative gene expression in bone samples. SOST, FGF23 and PHEX transcripts were measured by RT-qPCR in bone tissue samples from control individuals, patients with hip fractures (Frx) and hip osteoarthritis (OA). Bars represent mean and SD expression values relative to the housekeeping gene TBP in a $\log 2$ scale. $\mathrm{P}$ values of the differences across groups are also shown.

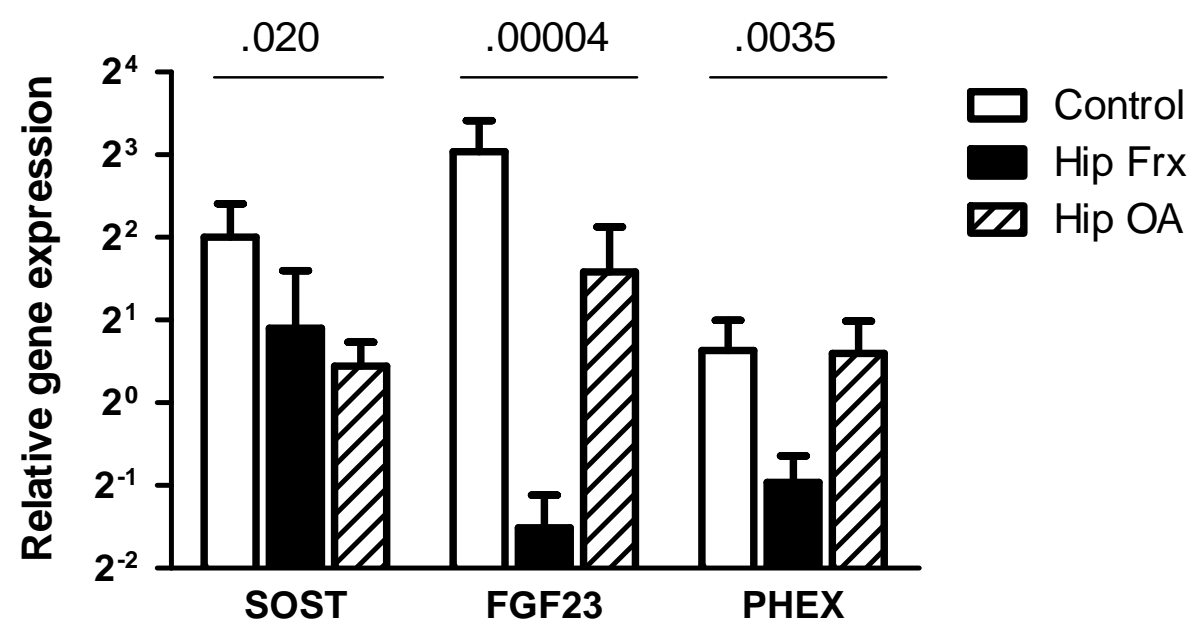


Fig. 3. Distance from sclerostin-positive and sclerostin-negative osteocytic lacunae to the closest bone surface in each group (mean and SE in control samples; hip fractures -Frx-, and hip osteoarthritis -OA). Black bars, sclerostin-positive; dashed bars, sclerostin-negative lacunae.

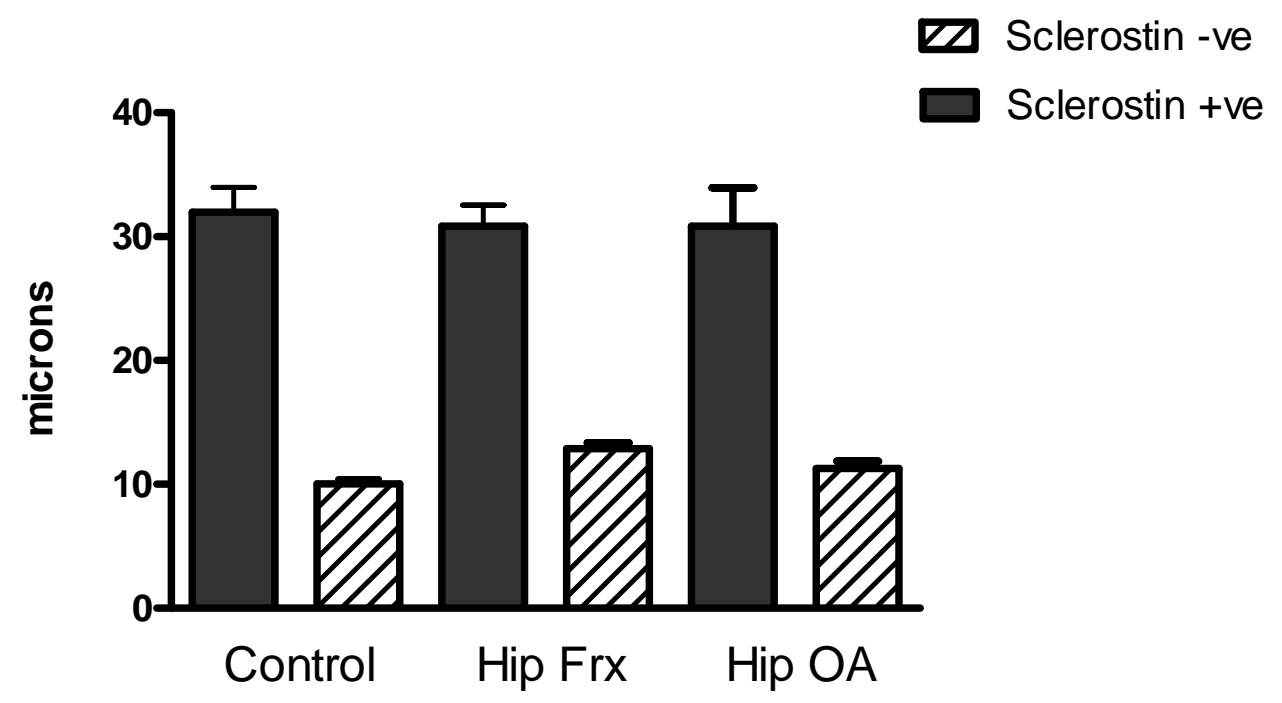


Fig. 4. Sclerostin-positive lacunae, occupied lacunae (ie, lacunae with an osteocyte) and lacunae with activated caspase 3 staining in bone samples of different groups. Graphs represent the mean and SE percentages over total number of lacunae. Representative sections stained with anti-sclerostin (top) or anti-caspase 3 antibody (bottom). The arrows mark some positive (black arrow) and negative (white arrow) lacunae. The middle panel shows a section stained only with hematoxylin; arrows mark an occupied (black) and an empty lacunae (white).
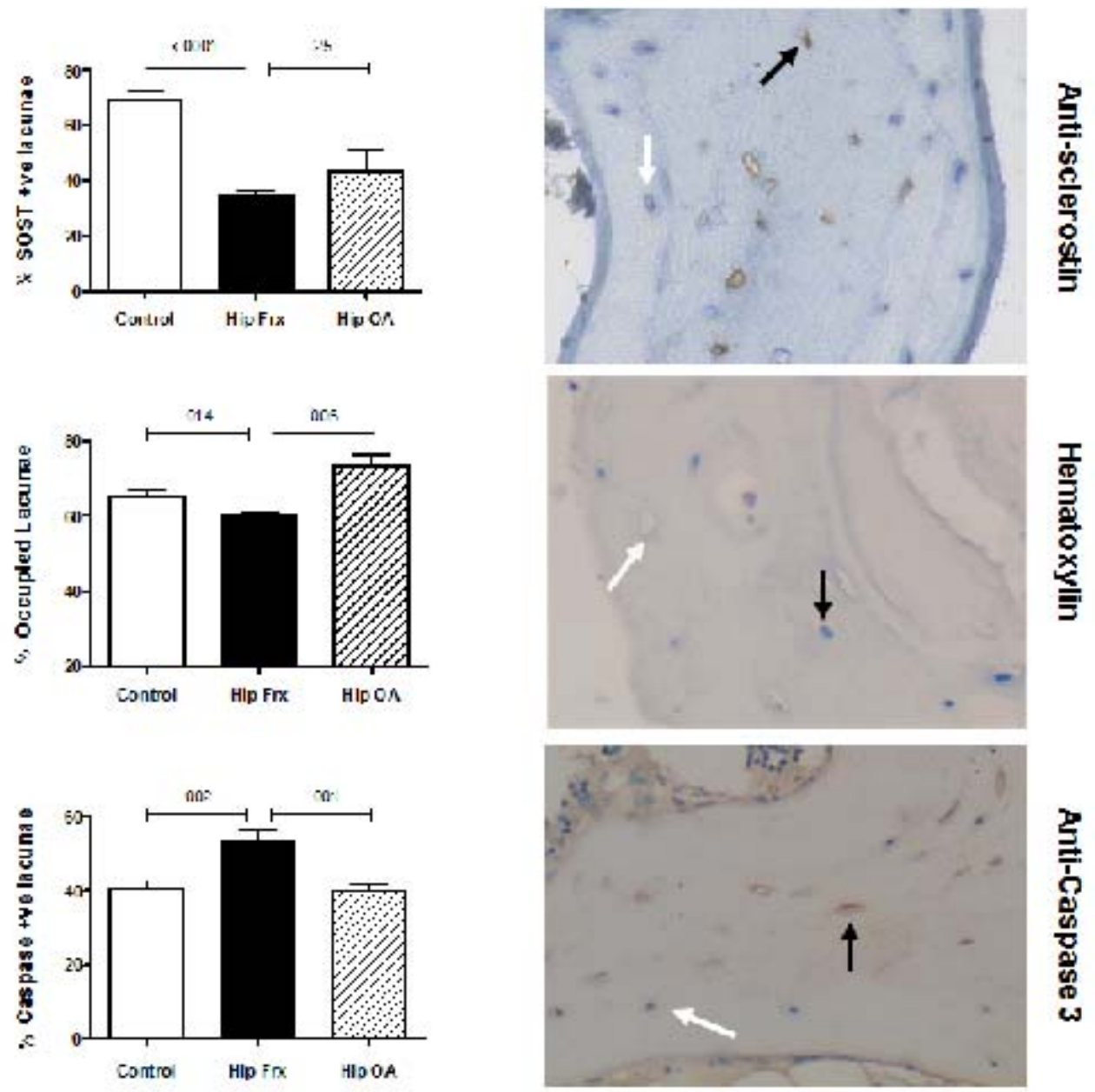
Fig 5. Multi-axis radial graph summarizing gene expression of SOST, FGF23 and PHEX; occupied lacunae (Ocy number); caspase-positive lacunae (Ocy apoptosis); and lacunae density in patients with hip fractures (continuous line) and hip osteoarthritis (dashed line). Results are expressed as percentages of the values in control bone samples, which are represented by the grey area.

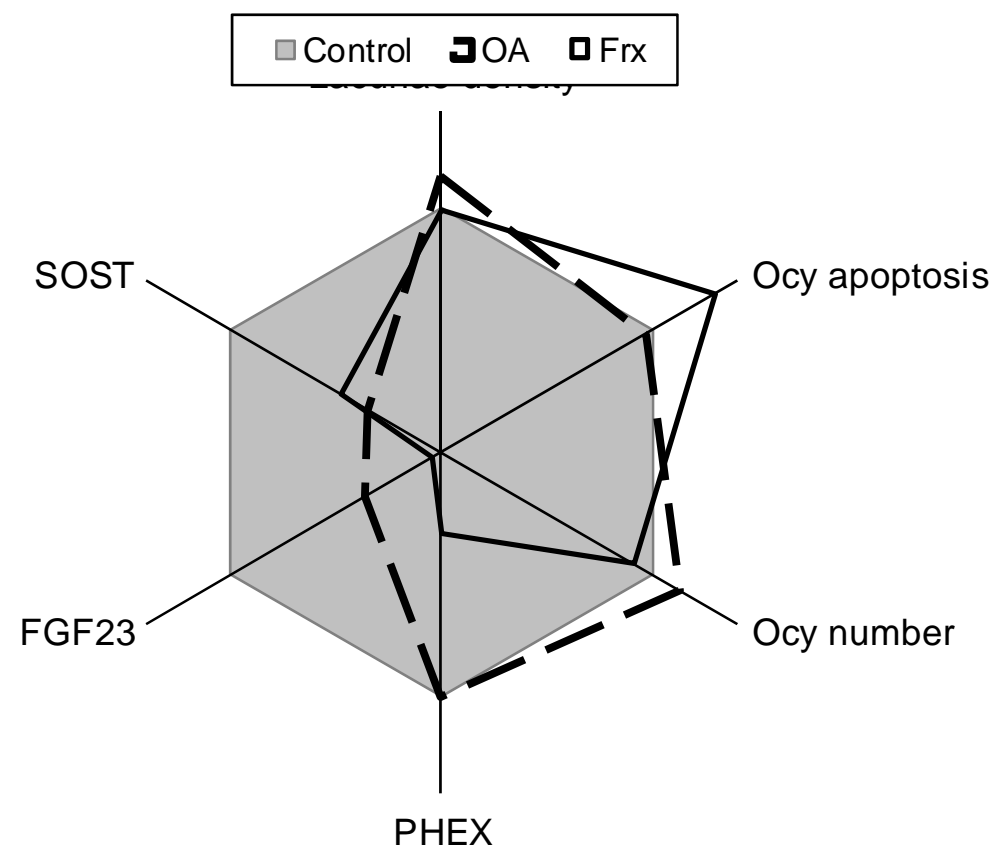

